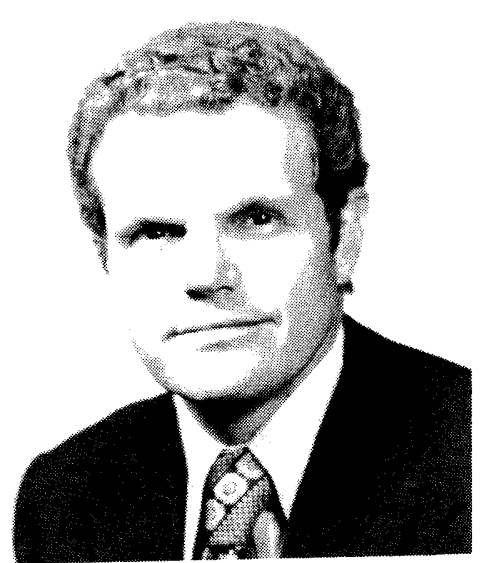

Area Co-Director:

Bruce Wrigley

Travelers Insurance

Hartford, Connecticut

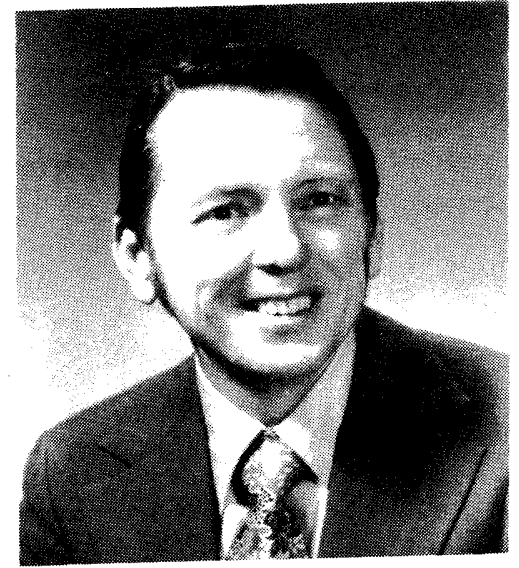

Area Co-Director: Edward J. Palmer Boston University

Boston, Massachusetts

\title{
User's viewpoint on EDP
}

Two prime concerns of the user of data processing equipment today are formalism in the administration of the DP function and the question of privacy. The former is becoming important through management pressures for engineering-like controls and procedures. Emphasis is being generated on the latter by activities of governmental bodies in proposing legislation which will control data processing practices. Both pressures will require rapid and definitive response. These concerns are addressed in a series of four panel discussions structured for the EDP user. Panelists discuss the issues and outline their solutions to some of the problems.

There have been advances in the state of the art of systems development recently, and even newer methodologies are being experimented with. In the session on "Benefits of New Programming Methodologies," the impact of these methods on the effectiveness of the organization is explored by those who have used them. One of the emerging trends in larger data processing organizations is the establishment of functional units devoted to the optimization of resource utilization. Methods, tools and results are discussed by individuals who have been deeply involved in this new activity in the session titled "Optimization of EDP Installations." There are many organizations currently involved in the selection process for data base management systems. There are very few guidelines to follow in this process and a formal technique for making such an analysis is strongly needed. A panel has been assembled for "Selection Techniques for Packaged Data Management Systems" consisting of individuals who have each used different formal methodologies resulting in the selection of different packages. The panelists will present their methodologies and argue the relative importance of various factors in such a selection. In the final session, "Issues and Answers-Data Security and Personal Privacy," some of the people who are active in the legislative process regarding privacy and security develop a picture in regard to what the user can expect in the near future, what is needed, and the implications of both to the data processing function.

No formal papers are included in this group of sessions. 
